\title{
Thermal noise from optical coatings in gravitational wave detectors
}

\author{
Gregory M. Harry, Helena Armandula, Eric Black, D. R. M. Crooks, Gianpietro Cagnoli, \\ Jim Hough, Peter Murray, Stuart Reid, Sheila Rowan, Peter Sneddon, Martin M. Fejer, \\ Roger Route, and Steven D. Penn
}

\begin{abstract}
Gravitational waves are a prediction of Einstein's general theory of relativity. These waves are created by massive objects, like neutron stars or black holes, oscillating at speeds appreciable to the speed of light. The detectable effect on the Earth of these waves is extremely small, however, creating strains of the order of $10^{-21}$. There are a number of basic physics experiments around the world designed to detect these waves by using interferometers with very long arms, up to $4 \mathrm{~km}$ in length. The next-generation interferometers are currently being designed, and the thermal noise in the mirrors will set the sensitivity over much of the usable bandwidth. Thermal noise arising from mechanical loss in the optical coatings put on the mirrors will be a significant source of noise. Achieving higher sensitivity through lower mechanical loss coatings, while preserving the crucial optical and thermal properties, is an area of active research right now. (C) 2006 Optical Society of America

OCIS codes: $310.1620,310.6870,350.1270$.
\end{abstract}

\section{Introduction}

Isaac Newton's description of gravity was improved upon in 1915 by Albert Einstein when the latter's general theory of relativity was published. This theory allows for oscillations in space-time, caused by motions of masses analogous to electromagnetic waves arising from moving charges in Maxwell's theory. These oscillations, known as gravitational waves, create a strain in space-time, so the travel time for a light beam between two inertial masses will change as the wave goes by. The size of this strain is set by the ratio

G. M. Harry (gharry@ligo.mit.edu), H. Armandula, and E. Black are with the Laser Interferometer Gravitational-Wave Observatory (LIGO) Laboratory; G. M. Harry, with the Massachusetts Institute of Technology, NW17-161, Cambridge, Massachusetts 01239; H. Armandula and E. Black, with the California Institute of Technology, 1200 East California Boulevard, Pasadena, California 91125. D. R. M. Crooks, G. Cagnoli, J. Hough, P. Murray, S. Reid, S. Rowan, and P. Sneddon are with the Department of Physics and Astronomy, The University of Glasgow, Glasgow G12 8QQ, United Kingdom. M. M. Fejer and R. Route are with the Edward L. Ginzton Laboratory, Stanford University, Stanford, California 94305. S. D. Penn is with the Department of Physics, Hobart and William Smith Colleges, Geneva, New York 14456.

Received 1 March 2005; accepted 7 July 2005.

0003-6935/06/071569-06\$15.00/0

(C) 2006 Optical Society of America

$$
G m v^{2} /\left(r c^{4}\right) \approx 10^{-21}\left(\frac{m}{M_{\odot}}\right)\left(\frac{v}{c}\right)^{2}\left(\frac{100 \mathrm{Mpc}}{r}\right),
$$

where $G$ is Newton's gravitational constant, $m$ is the mass of the source, $v$ is the velocity of the source, $r$ is the distance from the detector to the source, $c$ is the speed of light, $M_{\odot}$ is a solar mass, and Mpc is a megaparsec. To even approach measurable strains, astronomical-sized masses moving at appreciable fractions of the speed of light are necessary. A typical gravitational wave at Earth from a source at intergalactic distances is expected to have a strain near $10^{-21}$ or less. This is roughly a change in length equal to the width of a human hair over the distance between the Sun and the nearest star.

There are a number of experiments ${ }^{1-3}$ that use interferometry to attempt to detect these waves. A typical Michelson interferometer design with two perpendicular arms is shown in Fig. 1. The tensor field of the gravitational wave is most easily detected by using two perpendicular arms. This is in contrast to the vector electromagnetic field, in which a single linear antenna suffices. The mirrors and other optics of the interferometer hang as pendulums. This gives the best approximation of a freely falling mass; nearly free in the sensitive direction of the interferometer but supported against the static gravitational field of the Earth. To increase the signal, many experiments make each long arm a Fabry-Perot cavity to increase the interaction time with the mirrors. 


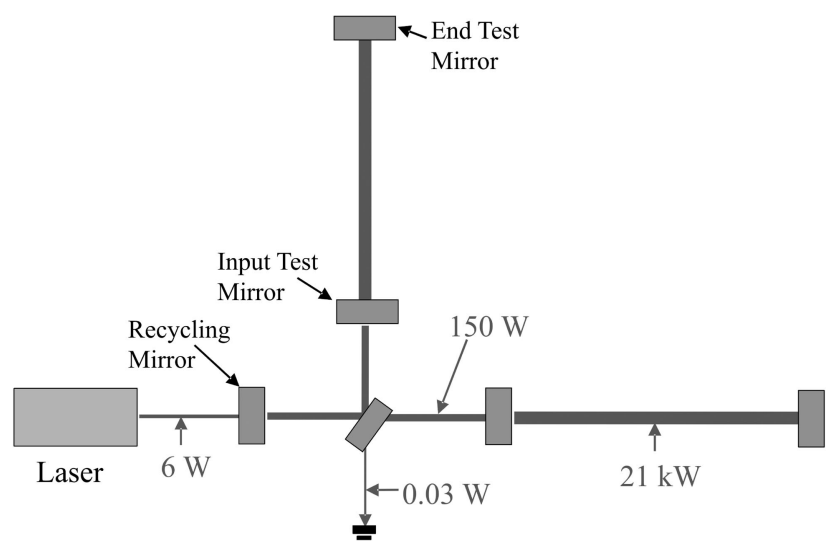

Fig. 1. Schematic drawing of a gravitational wave interferometer. The laser on the left puts light into the system, which then passes the power recycling mirror and is then divided by the beam splitter into two beams going to each arm. The arms are Fabry-Perot cavities formed by an input test mirror and an end test mirror. The light power in each cavity is shown.

The entire path of the laser and the optics are in vacuum to minimize optical path-length fluctuations, among other reasons. The interferometer is sensitive to changes in the position of the mirrors, so longer arms translate the same strain into larger displacements. The longest interferometers have arms $4 \mathrm{~km}$ long, so the typical gravitational wave strain will create approximately $10^{-18} \mathrm{~m}$ of mirror motion. Keeping position and sensing noise down thus becomes a crucial design criterion.

LIGO (Laser Interferometer Gravitational Wave Observatory) is the American-funded project to detect gravitational waves. LIGO has two observatories, one in Livingston, Louisiana (east of Baton Rouge), and the other in eastern Washington state on the Department of Energy Hanford Reservation. These sites are shown in Fig. 2. Livingston has a single interferometer with $4 \mathrm{~km}$ long arms, Hanford has two interferometers, one with $4 \mathrm{~km}$ arms and one half-length with $2 \mathrm{~km}$ arms, both within a single vac- uum system. Other detectors in Germany (GEO 600), Italy (Virgo), and Japan (TAMA 300) complement the LIGO interferometers and form an international network. It is possible that these first-generation instruments will detect a gravitational wave, but it is likely that higher-sensitivity instruments will be necessary. The expected sensitivity of the planned Advanced LIGO should be sufficient to detect gravitational waves as often as once a day. ${ }^{4}$

\section{LIGO Gravitational Wave Detectors}

Here we give a brief overview of the design and performance of the operating LIGO interferometers. For a more detailed description, please see Ref. 1. Each LIGO detector is a Michelson interferometer with arms $4 \mathrm{~km}$ (or $2 \mathrm{~km}$ ) long. Each arm consists of a Fabry-Perot cavity with a finesse near 100. Optical power in the arms is further increased by including an additional optic, the power recycling mirror, ${ }^{5}$ at the bright port of the interferometer. The design for Advanced LIGO adds a further optic at the dark port, the signal recycling mirror, ${ }^{5}$ to give additional sensitivity in a band around a frequency of interest. This technique, known as signal recycling, is currently in use on the GEO 600 interferometer.

Measured noise from the current $4 \mathrm{~km}$ long interferometer at Hanford is shown in Fig. 3, along with the design goal. Every optic is isolated from ground motion by a multistage vibration-isolation structure, which reduces seismic noise that dominates the noise budget at frequencies below $\sim 40 \mathrm{~Hz}$. Thermal noise from the pendulum suspension sets the fundamental limit at intermediate frequencies. At higher frequencies, above $\sim 150 \mathrm{~Hz}$, shot noise is dominant.

Figure 3 also shows a possible noise budget for the proposed Advanced LIGO interferometer. Improved vibration-isolation stacks will move the frequency band to where seismic noise dominates down to $10 \mathrm{~Hz}$ and below. The added signal recycling mirror combines the shot noise and radiation pressure on the mirrors into a single, optical noise, which is dominant
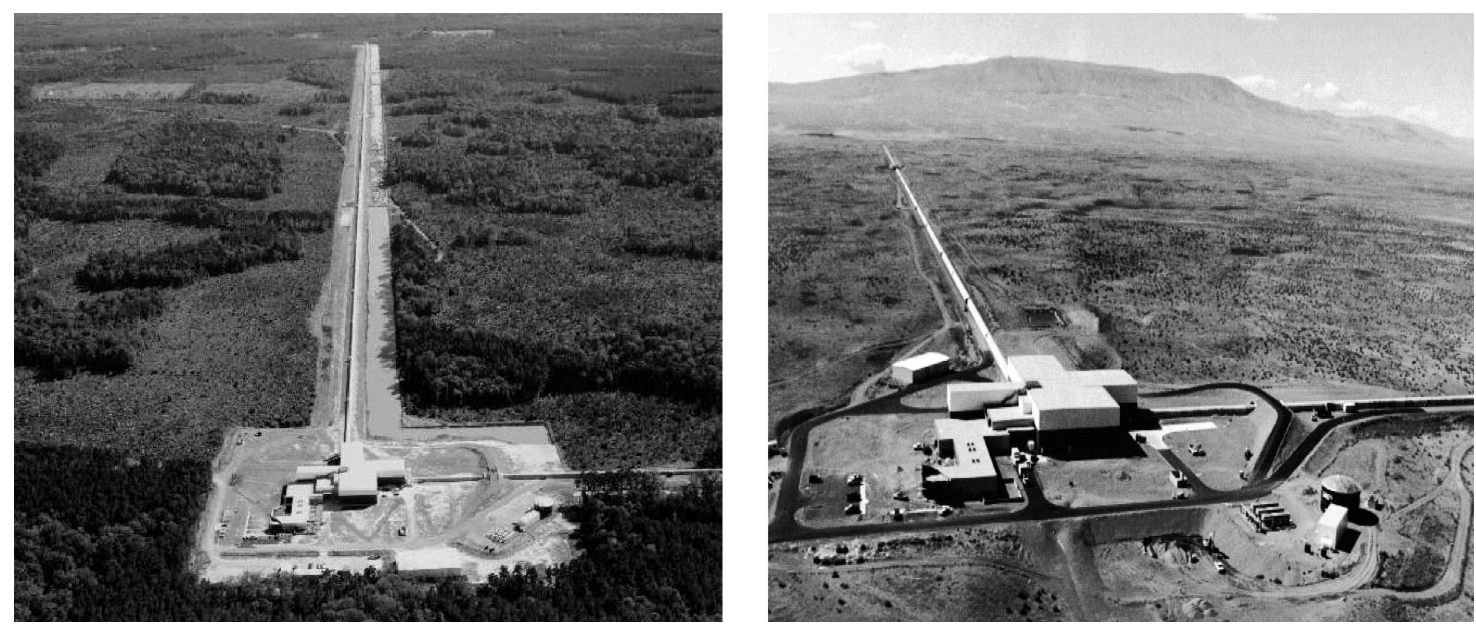

Fig. 2. Photographs of the LIGO sites: left, Livingston, Louisiana; right, Hanford, Washington. Each arm reaches $4 \mathrm{~km}$ from the building at the corner. 

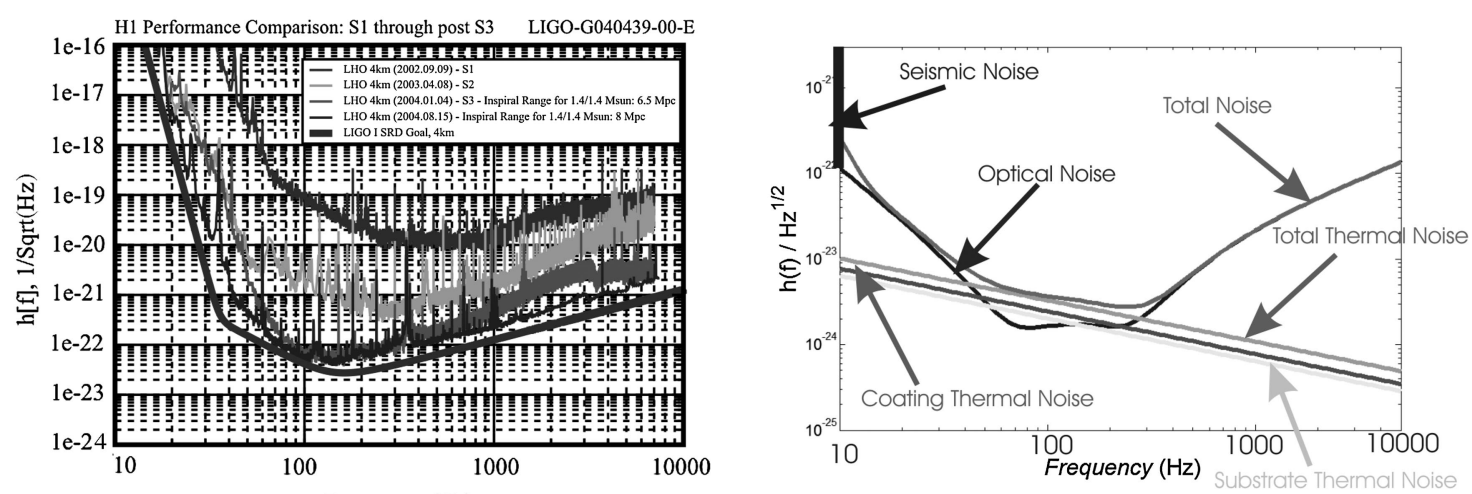

Fig. 3. Noise for the $4 \mathrm{~km}$ long LIGF interferometer in Hanford (left). The solid curve is the design noise; the curves above that show the measured noise from September 2002 to August 2004. The design sensitivity of the proposed Advanced LIGO interferometer is shown on the right. Note the difference in the $y$-axis offset between the two graphs; the Advanced LIGO will have lower noise (about a factor of 15) than the initial LIGO in most bands.

over most of the sensitive bandwidth. A higher-power laser will reduce shot noise at high frequencies. The interaction time with the mirrors will also be made higher by increasing the finesse of the Fabry-Perot cavities. In the most sensitive bandwidth, between $\sim 40 \mathrm{~Hz}$ and a few hundred hertz, thermal noise is the dominant noise source. This is the thermal motion of the mirror faces themselves and comes primarily from the mechanical loss in the optical coatings. The thermal-noise curve in Fig. 3 assumes the same ionbeam-deposited silica/tantala coating as was used for the initial LIGO. This noise will set the ultimate sensitivity, and thus the astronomical effectiveness, of the Advanced LIGO interferometers. Reducing this noise from the level shown would have big payoffs for gravitational wave detection and astronomy.

\section{Coating Thermal Noise}

Thermal noise is caused by mechanical loss in accordance with the fluctuation-dissipation theorem. ${ }^{6}$ Direct application of this theorem to the case of a Gaussian-profile laser sensing the position of a coated mirror, as in a gravitational wave interferometer, yields ${ }^{7}$

$$
S_{x}(f)=2 k_{B} T \phi_{\text {eff }}(1-\sigma) /\left(\pi^{3 / 2} f w Y\right)
$$

for the thermal noise. Here $S_{x}(f)$ is the power spectral density of position noise, $k_{B}$ is Boltzmann's constant, $T$ is the temperature, $\sigma$ is the Poisson ratio of the substrate material, $w$ is the half-width of the Gaussian laser beam, and $\phi_{\text {eff }}$ is the effective loss angle of the mirror, given by

$$
\begin{aligned}
\phi_{\mathrm{eff}}= & \phi+d /\left(\sqrt{\pi} w Y_{\perp}\right)\left(\left\{Y /\left(1-\sigma_{\perp}\right)-2 \sigma_{\perp}^{2} Y Y_{\|} /\right.\right. \\
& {\left.\left[Y_{\perp}\left(1-\sigma^{2}\right)\left(1-\sigma_{\|}\right)\right]\right\} \phi_{\perp}+Y_{\|} \sigma_{\perp}(1-2 \sigma) / } \\
& {\left[\left(1-\sigma_{\|}\right)(1-\sigma)\right]\left(\phi_{\|}-\phi_{\perp}\right)+Y_{\|} Y_{\perp}(1+\sigma) } \\
& \left.\times(1-2 \sigma)^{2} /\left[Y\left(1-\sigma_{\|}^{2}\right)(1-\sigma)\right] \phi_{\|}\right),
\end{aligned}
$$

where $d$ is the coating thickness and $Y, \sigma$, and $\phi$ are the Young's moduli, the Poisson's ratios, and the loss angles of the silica substrate (no subscript) and the coating for stresses perpendicular $(\perp)$ and parallel $(\|)$ to the optic face, respectively. If all the Poisson ratios are small, Eq. (2) can be approximated as

$$
\phi_{\mathrm{eff}} \approx \phi+d /(\sqrt{\pi} w)\left(Y / Y_{\perp} \phi_{\perp}+Y_{\|} / Y \phi_{\|}\right) .
$$

The elastic constants of the coating can be calculated from the bulk values of the individual materials that make up the coating (silica and tantala in the current LIGO mirrors). For a coating made of alternating layers of two dielectrics, these values are

$$
\begin{aligned}
Y_{\perp} & =\left(d_{1}+d_{2}\right) /\left(d_{1} / Y_{1}+d_{2} / Y_{2}\right), \\
Y_{\|} & =\left(Y_{1} d_{1}+Y_{2} d_{2}\right) /\left(d_{1}+d_{2}\right), \\
\sigma_{\perp} & =\left(\sigma_{1} Y_{1} d_{1}+\sigma_{2} Y_{2} d_{2}\right) /\left(Y_{1} d_{1}+Y_{2} d_{2}\right), \\
\phi_{\perp} & =Y_{\perp}\left(\phi_{1} d_{1} / Y_{1}+\phi_{2} d_{2} / Y_{2}\right), \\
\phi_{\|} & =\left(Y_{1} \phi_{1} d_{1}+Y_{2} \phi_{2} d_{2}\right) /\left(Y_{\|}\left(d_{1}+d_{2}\right)\right),
\end{aligned}
$$

where subscripts 1 and 2 refer to the two materials. For $\sigma_{\|}$the equivalent equation is more complicated. The average of the two material's Poisson ratios agrees with a numerical solution to within 5\% for the case of a $\mathrm{SiO}_{2} / \mathrm{Ta}_{2} \mathrm{O}_{5}$ coating with thicknesses appropriate for high reflectivity at $1.064 \mu \mathrm{m}$, the laser wavelength.

This model assumes that the thermal noise arises from internal friction in the coating and substrate. Thermoelastic loss, ${ }^{8}$ in which expansion and contraction of an optic is connected with heat flow between the coating and the substrate ${ }^{9,10}$ or within the substrate, ${ }^{11}$ can also be a source of thermal noise. To minimize thermoelastic loss between the coating and the substrate, one should ensure that thermal expansion coefficients and Young's moduli are matched. Matching Young's moduli will also help reduce thermal noise from internal friction, as seen in Eq. (4), as long as the loss angles are similar for stresses parallel 
Table 1. Requirements for the Advanced LIGO Coating ${ }^{a}$

\begin{tabular}{lcc}
\hline \multicolumn{1}{c}{ Parameter } & $\begin{array}{c}\text { Advanced LIGO } \\
\text { Requirement }\end{array}$ & $\begin{array}{c}\text { Demonstrated } \\
\text { Value }\end{array}$ \\
\hline Loss angle $\phi_{\|}$ & $5 \times 10^{-5}$ & $1.5 \times 10^{-4}$ \\
Optical absorption & $0.5 \mathrm{ppm}$ & $1 \mathrm{ppm}$ \\
Scatter & $2 \mathrm{ppm}$ & $20 \mathrm{ppm}$ \\
Thickness uniformity & $10^{-3}$ & $8 \times 10^{-3}$ \\
Transmission & $5 \mathrm{ppm}$ & $5.5 \mathrm{ppm}$ \\
Transmission matching & $5 \times 10^{-3}$ & $1 \times 10^{-2}$ \\
\hline
\end{tabular}

${ }^{a}$ These values are necessary to reach the high cavity finesse and low thermal noise needed. The demonstrated values are not all shown on a single coating. In addition, the thermal expansion coefficient and Young's modulus of the coating must be matched to that of the substrate within a factor of $\sim 3$.

and perpendicular to the face. It is expected that acceptably good matching between a coating and a silica substrate can be accomplished, so the limiting thermal noise will be dominated by the internal friction in the coating.

\section{Coating Research for Advanced LIGO}

The Advanced LIGO coatings must satisfy strict optical as well as mechanical and thermal properties. High reflectivity is needed to create high-finesse Fabry-Perot arm cavities. Table 1 shows the coating requirements along with the best demonstrated value, either in an initial LIGO interferometer or a laboratory research sample. Ideally, the Young's moduli and thermal expansion coefficients should be matched between the coating and the substrate as well. Research on developing an acceptable coating for the Advanced LIGO has focused on reducing the internal friction owing to the importance of thermal noise to the noise budget, the lack of information about internal friction in dielectric coatings, and the fact that optical loss (absorption, scatter, and transmission) can be made up for by higher laser power.
Higher laser power is limited, however, by the ability to control the heating of the optics, ${ }^{12}$ among other reasons. Coating thermal noise is also the limiting noise source for laser frequency stabilization, ${ }^{13}$ making this research effort important for other precision experiments that use interferometry.

The mechanical loss in the coatings is being studied by measuring the ringdown time of normal modes of silica disks coated with target coatings. The quality factors of normal modes are measured before and after coating to determine its effect. Two geometries are being used: thin samples $(0.25 \mathrm{~cm}$ thick $)$ and thick samples (2.5 cm thick), both $7.6 \mathrm{~cm}$ in diameter. The two geometries allow normal modes between approximately 2.7 and $21 \mathrm{kHz}$ to be measured. Wire loops are used to suspend the thick samples from a rigid structure, while the thin samples hang below welded silica fibers. Both samples hang in vacuum to eliminate any friction due to the air. The normal modes are rung up by using a comb capacitor and are monitored by using either a birefringence readout or a simple Michelson interferometer. Figure 4 shows a schematic of both experimental setups, and more details are given in recently published papers. ${ }^{14,15}$

The loss angle of the coating, $\phi_{\|}$, is determined from the measured modal $Q$ 's by

$$
1 / Q_{\text {coated }}=1 / Q_{\text {uncoated }}+\phi_{\|} E_{\text {coating }} / E_{\text {total }}
$$

where $Q_{\text {coated }}$ is the measured $Q$ of the coated sample, $Q_{\text {uncoated }}$ is the measured $Q$ of the uncoated sample, $E_{\text {coating }}$ is the total elastic energy stored in the coating for the given mode, $E_{\text {total }}$ is the total elastic energy in the mode over the whole sample, and only $\phi_{\|}$of the coating enters because the free-boundary condition on the surface of the sample requires that there be no stress perpendicular to the face.

A first round of measurements was made to determine the source of mechanical loss in the silica/

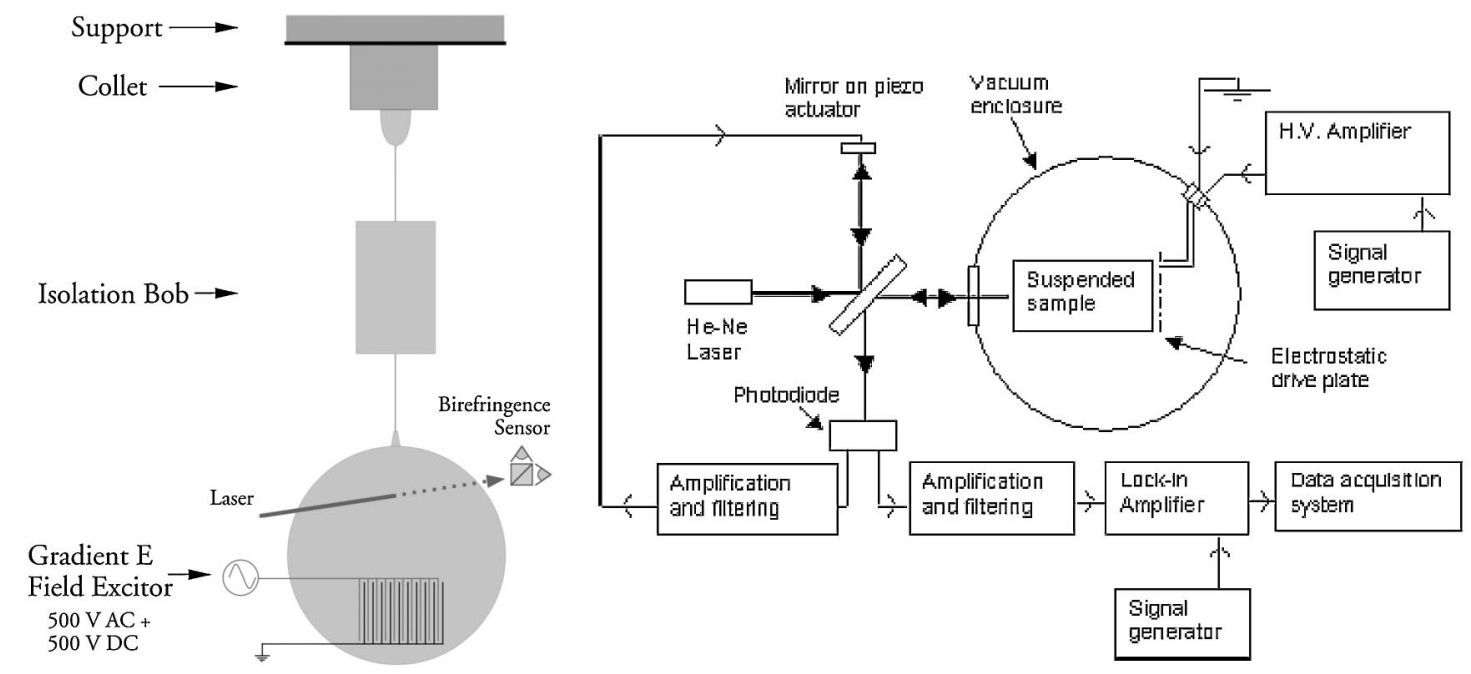

Fig. 4. Schematic of the thin-disk experimental setup is presented on the left, showing the welded silica suspension and birefringence readout. A schematic of the thick-disk experimental setup is presented on the right, showing the interferometer readout and related electronics. 
Table 2. Results of the First Round of the Mechanical Loss Experiments ${ }^{a}$

\begin{tabular}{cccc}
\hline $\begin{array}{c}\text { Total } \\
\text { Layers }\end{array}$ & $\begin{array}{c}\mathrm{SiO}_{2} \text { Optical } \\
\text { Thickness }\end{array}$ & $\begin{array}{c}\mathrm{Ta}_{2} \mathrm{O}_{5} \text { Optical } \\
\text { Thickness }\end{array}$ & Loss Angle $\phi_{\|}$ \\
\hline 2 & $\lambda / 4$ & $\lambda / 4$ & $2.7 \pm 0.7 \times 10^{-4}$ \\
30 & $\lambda / 4$ & $\lambda / 4$ & $2.6 \pm 0.7 \times 10^{-4}$ \\
60 & $\lambda / 8$ & $\lambda / 8$ & $2.7 \pm 0.5 \times 10^{-4}$ \\
30 & $\lambda / 8$ & $3 \lambda / 8$ & $3.7 \pm 0.5 \times 10^{-4}$ \\
30 & $3 \lambda / 8$ & $\lambda / 8$ & $1.9 \pm 0.2 \times 10^{-4}$ \\
\hline
\end{tabular}

${ }^{a}$ The goal was to determine the source of loss in $\mathrm{SiO}_{2} / \mathrm{Ta}_{2} \mathrm{O}_{5}$ coatings.

tantala coatings. Three different sources were considered; internal friction in the silica and tantala, interfacial rubbing between the coating and the substrate, and rubbing between the individual layers of the multilayer coating. Both thin and thick samples were coated with each coating, with all coating performed by SMA/Virgo (Now LMA/Virgo) in Lyon, France. The first samples had only a two-layer coating, one layer each of silica and tantala. This was to test for rubbing between the coating and the substrate. The second set had 30 layers of alternating silica and tantala to use as a baseline for comparisons. The third set had 60 layers of silica and tantala, but each layer was only half as thick as those in the 30-layer set, making the whole coating the same thickness. This tests for an effect from the coating layer interfaces. The fourth set had thicker tantala layers, but thinner silica, so the coating was the same thickness as the baseline. The fifth had thicker silica with thinner tantala. These two test whether it is internal friction in either the silica or tantala that dominates the coating mechanical loss. The results of these experiments are presented in Table 2. A more thorough explanation of this study is given in Ref. 16 .

The results in Table 2 show that internal friction is the cause of the mechanical loss in the coatings, and it is the internal friction of the tantala that is the primary source. Using the results from the three sets with differing material thicknesses, one can determine the loss angles for the silica and tantala:

$$
\begin{gathered}
\phi_{\mathrm{SiO}_{2}}=0.5 \pm 0.3 \times 10^{-4}, \\
\phi_{\mathrm{Ta}_{2} \mathrm{O}_{5}}=4.4 \pm 0.2 \times 10^{-4} .
\end{gathered}
$$

The results in Eqs. (11) and (12) assume a structure (i.e., frequency independent) model for mechanical loss.

Samples coated by MLD Technologies of Mountain View, California, with 30 layers of $\lambda / 4$ alternating silica and tantala were also measured, giving results similar to those in Table 2. Additional measurements were also made on alumina/tantala. The preliminary analysis is consistent with the result that internal friction of the tantala is the dominant source of loss in the alumina/tantala coating as well.

Examining the mechanical loss in the coatings mode by mode allows for the frequency dependence to be explored. Using a model with a frequencyindependent term and a term proportional to frequency yields ${ }^{17}$

$$
\begin{gathered}
\phi_{\mathrm{SiO}_{2}}=(0.4 \pm 0.3) \times 10^{-4}+f(2.7 \pm 0.9) \times 10^{-9}, \\
\phi_{\mathrm{Ta}_{2} \mathrm{O}_{5}}=(4.2 \pm 0.4) \times 10^{-4}+f(0.4 \pm 0.9) \times 10^{-9},
\end{gathered}
$$

where $f$ is in hertz. However, these results depend heavily on a few modal $Q$ results at low frequency. Research is in progress to measure more modes of the thin samples to allow the frequency space between the modes of thin and thick samples to be more fully measured.

Direct measurement of thermal noise from silica/ tantala coatings has been accomplished both in Japan $^{18}$ and at Caltech by LIGO's thermal noise interferometer (TNI). ${ }^{19}$ The TNI consists of a Michelson interferometer with Fabry-Perot arms similar to those of LIGO, but with arm lengths of $1 \mathrm{~cm}$ rather than $4 \mathrm{~km}$. The shorter arms result in a smaller spot size, which as seen in Eq. (4) means that the thermal noise will be higher. The TNI has a spot size of $0.15 \mathrm{~mm}$, compared with $40 \mathrm{~mm}$ in the initial LIGO and $60 \mathrm{~mm}$ planned for the Advanced LIGO. The TNI used mirrors coated with $4.26 \mu \mathrm{m}$ of a silica/tantala coating prepared by Research Electro-Optics of Boulder, Colorado. Noise data from the TNI are shown in Fig. 5. These data show the expected $1 / \sqrt{f}$ frequency-dependent characteristic of thermal noise (with structural damping) and are consistent with loss angles for the coating of $\phi_{\perp}=1.2 \times 10^{-4}$ and $\phi_{\|}=4.2 \times 10^{-4}$, or $\phi_{\mathrm{Ta}_{2} \mathrm{O}_{5}}=5.1 \times 10^{-4}$ assuming $\phi_{\mathrm{SiO}_{2}}=5 \times 10^{-5}$.

Further research is ongoing to reduce the loss angle of the coating, focusing on the tantala. In collaboration with LMA/Virgo, a titania dopant of various concentrations was added to the tantala in a number of test coatings. Preliminary results from these samples are shown in Table 3. Increasing concentrations

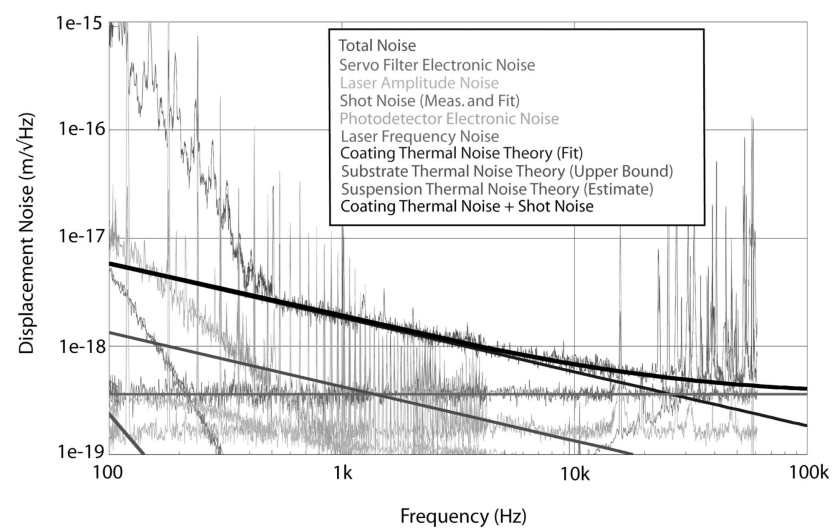

Fig. 5. Direct measurement of coating thermal noise by LIGO's thermal noise interferometer (TNI). Direct measurements allow a check on the inferred coating loss angles from ringdown measurements. The noise between $500 \mathrm{~Hz}$ and $6 \mathrm{kHz}$ is due to thermal noise from the coating. 
Table 3. Mechanical Loss of $\mathrm{SiO}_{2} / \mathrm{TiO}_{2}$-Doped $\mathrm{Ta}_{2} \mathrm{O}_{5}$ Coatings from LMA/Virgo ${ }^{a}$

\begin{tabular}{cc}
\hline $\begin{array}{c}\text { Concentration } \\
\text { of } \mathrm{TiO}_{2}\end{array}$ & Loss Angle $\phi_{\|}$ \\
\hline None & $2.7 \pm 0.5 \times 10^{-4}$ \\
Low & $1.8 \pm 0.2 \times 10^{-4}$ \\
High & $1.6 \pm 0.2 \times 10^{-4}$ \\
\hline
\end{tabular}

${ }^{a}$ All coatings were composed of 30 layers, with $\lambda / 4$ optical thickness for each layer.

of titania are seen to lower the mechanical loss. These titania-doped coatings have only $\sim 1 \mathrm{ppm}$ (parts per million) of optical absorption, so they are close to meeting the Advanced LIGO's optical specifications.

There are other ideas for reducing coating thermal noise in advanced interferometers. One is to use corner reflectors, rather than optics coated on a face. ${ }^{20} \mathrm{An}$ other is to have an additional short cavity in place of the current end test masses, with each mirror of this cavity having a coating thinner than the baseline design. ${ }^{21}$ Both of these ideas would require further research before being implemented on an operating detector.

\section{Conclusion}

Detection of gravitational waves is one of the great challenges in modern experimental physics. Reducing noise in the interferometers is the primary hurdle that must be overcome. Next-generation detectors will have their sensitivity limited by thermal noise from the optical coatings. Developing optical coatings with low mechanical loss to reduce the thermal noise while maintaining high reflectivity, low absorption, and low scatter is an important area of research in the experimental gravity community.

Progress has been made with reducing mechanical loss, but more work remains to be done. Technical input from all those with knowledge about coatings and optics will be crucial to achieving this goal. The LIGO Scientific Collaboration is interested in forging collaborations with researchers who have experience and knowledge in this area.

The LIGO Observatories were constructed by the California Institute of Technology and the Massachusetts Institute of Technology with funding from the National Science Foundation under cooperative agreement PHY-9210038. The LIGO Laboratory operates under cooperative agreement PHY-0107417. This paper was assigned LIGO document number LIGO-P040033-00-D.

\section{References}

1. B. Abbott and The LIGO Science Collaboration, "Detector description and performance for the first coincidence observations between LIGO and GEO," Nucl. Instrum. Methods Phys. Res. A 517, 154-179 (2004).

2. F. Acernese and the VIRGO Collaboration, "Status of VIRGO," Class. Quantum Grav. 19, 1421-1428 (2002).

3. M. Ando and the TAMA Collaboration, "Current status of TAMA," Class. Quantum Grav. 19, 1409-1419 (2002).

4. K. S. Thorne and C. Cutler, "An overview of gravitational wave sources," in Proceedings of 16th International Conference on General Relativity and Gravitation, N. T. Bishop and S. D. Maharaj, eds. (World Scientific, 2002), pp. 72-112.

5. B. J. Meers, "Recycling in a laser-interferometric gravitationalwave detector," Phys. Rev. D 38, 2317-2326 (1988).

6. H. B. Callen and R. F. Greene, "On a theorem of irreversible thermodynamics," Phys. Rev. 86, 702-710 (1952).

7. Yu. Levin, "Internal thermal noise in the LIGO test masses: a direct approach,” Phys. Rev. D 57, 659-663 (1998).

8. C. Zener, "Internal friction in solids. I. Theory of internal friction in reeds," Phys. Rev. 52, 230-235 (1937).

9. M. M. Fejer, S. Rowan, G. Cagnoli, D. R. M. Crooks, A. Gretarsson, G. M. Harry, J. Hough, S. D. Penn, P. H. Sneddon, and S. P. Vyatchanin, "Thermoelastic dissipation in inhomogeneous media: loss measurements and displacement noise in coated test masses for interferometric gravitational wave detectors," Phys. Rev. D 70, 082003 (2004).

10. V. B. Braginsky and S. P. Vyatchanin, "Thermodynamical fluctuations in optical mirror coatings," Phys. Lett. A 312, 224 255 (2003).

11. V. B. Braginsky, M. L. Gorodetsky, and S. B. Vyatchanin, "Thermodynamical fluctuations and photo-thermal shot noise in gravitational wave antennae," Phys. Lett. A 264, 1-10 (1999).

12. R. Lawrence, D. Ottaway, P. Fritschel, and M. Zucker, "Active correction of beam heating induced phase distortions in optics via external radiative thermal acutation," Opt. Lett. 29, 26352637 (2004).

13. K. Numata, A. Kemery, and J. Camp, "Thermal-noise limit in the frequency stabilization of lasers with rigid cavities," Phys. Rev. Lett. 93, 250602 (2004).

14. G. M. Harry, A. M. Gretarsson, P. R. Saulson, S. E. Kittelberger, S. D. Penn, W. J. Startin, S. Rowan, M. M. Fejer, D. R. M. Crooks, G. Cagnoli, J. Hough, and N. Nakagawa, "Thermal noise in interferometric gravitational wave detectors due to dielectric optical coatings," Class. Quantum Grav. 19, 897-917 (2002).

15. D. R. M. Crooks, P. Sneddon, G. Cagnoli, J. Hough, S. Rowan, M. M. Fejer, E. Gustafson, R. Route, N. Nakagawa, D. Coyne, G. M. Harry, and A. M. Gretarsson, "Excess mechanical loss associated with dielectric mirror coatings on test masses in interferometric gravitational wave detectors," Class. Quantum Grav. 19-5, 883-896 (2002).

16. S. D. Penn, P. H. Sneddon, H. Armandula, Betzweiser, G. Cagnoli, J. Camp, D. R. M. Crooks, M. M. Fejer, A. M. Gretarsson, G. M. Harry, J. Hough, S. E. Kittelberger, M. J. Mortonson, R. Route, S. Rowan, and C. C. Vassiliou, "Mechanical loss in tantala/silica dielectric mirror coatings," Class. Quantum Grav. 20, 2917-2928 (2003).

17. D. R. M. Crooks, G. Cagnoli, M. M. Fejer, A. M. Gretarsson, G. M. Harry, J. Hough, N. Nakagawa, S. Penn, R. Route, S. Rowan, and P. H. Sneddon, "Experimental measurements of coating mechanical loss factors," Class. Quantum Grav. 21, S1059-S1065 (2004).

18. K. Numata, M. Ando, K. Yamamoto, S. Otsuka, and K. Tsubono, "Wide-band direct measurement of thermal fluctuations in an interferometer," Phys. Rev. Lett. 91, 260602 (2003).

19. E. D. Black, A. Villar, K. Barbary, A. Bushmaker, J. Heefner, S. Kawamura, F. Kawazoe, L. Matone, S. Meidt, S. R. Rao, K. Schulz, M. Zhang, and K. G. Libbrecht, "Direct observation of broadband coating thermal noise in a suspended interferometer," Phys. Lett. A 328, 1-5 (2004).

20. V. B. Braginsky and S. P. Vyatchanin, "Corner reflectors and quantum-non-demolition measurements in gravitational wave antennae," Phys. Lett. A 324, 345-360 (2004).

21. F. Ya. Khalili, "Reducing the mirrors coating noise in laser gravitational-wave antennae by means of double mirrors," Phys. Lett. A 334, 67-72 (2005). 